\title{
Perfil Nutricional de Crianças Portadoras de Tumores Cerebrais com Síndrome Diencefálica ou Síndrome de Russell recebendo Dieta Enteral
}

doi: https://doi.org/10.32635/2176-9745.RBC.2018v64n3.36

\author{
Nutritional Profile of Children with Brain Tumor with Diencephalic Syndrome or Russell Syndrome receiving Enteral Diet \\ Perfil Nutricional de Niños Portadores de Tumores Cerebrales con Síndrome Diencefálico o Síndrome de Russell \\ recibiendo Dieta Enteral
}

\author{
Adriana Garófolo'; Nasjla Saba Silva²; Sérgio Cavalheiro ${ }^{3}$
}

Resumo

Introduçáo: A síndrome diencefálica é uma doença pediátrica rara, decorrente de tumores hipotalâmicos, caracterizada por failure to thrive. Objetivo: Descrever o estado nutricional e a terapia nutricional por meio de sonda nasoenteral de pacientes com tumores cerebrais com a síndrome diencefálica. Método: Sete pacientes foram acompanhados de julho/1999 a abril/2002 e analisados retrospectivamente, usando os escores-z de peso para idade $(\mathrm{P} / \mathrm{I})$, peso para estatura $(\mathrm{P} / \mathrm{E})$ e estatura para idade $(\mathrm{E} / \mathrm{I})$ no diagnóstico da desnutrição. Todos foram avaliados por meio de composição corporal: prega cutânea triciptal (PCT) e circunferências do braço e muscular do braço (CB e $\mathrm{CMB}$ ) e receberam alimentação por sonda nasoenteral ou gastrostomia após o diagnóstico da neoplasia. Resultados: A idade variou de 2 meses a 13 anos, cinco do sexo masculino. A duração média da nutrição enteral foi de 7 meses $(1,1-18,5)$ após o diagnóstico, sem diferença estatística significante na evolução dos escores-z, apesar do aumento nas médias de P/I (-4,42 para -3,50) e P/E (-3,06 para -1,99), e dos indicadores de composição corporal (PCT: 2,85 para 4,88; CB: 9,81 para 11,84 e CMB: 8,91 para 10,31). Houve redução na média da E/I, caracterizando o atraso no crescimento dessas crianças. Conclusáo: A nutrição enteral demonstrou garantir a oferta nutricional e recuperar em parte os indicadores nutricionais de desnutriçáo aguda; principalmente a gordura corporal, mais do que massa magra. Entretanto, manteve-se o déficit de crescimento, agravado na maioria dos casos. A terapia nutricional deve ser implantada durante o tratamento oncológico, assegurando sua continuidade.

Palavras-chave: Síndrome de Silver-Russell; Neoplasias Encefálicas; Nutrição Enteral; Terapia Nutricional; Criança.

\begin{abstract}
Introduction: Diencephalic syndrome is a rare disorder of infancy characterized by profound emaciation with failure to thrive. The majority of cases of the syndrome are due to low grade gliomas of the anterior hypothalamus or optic nerve. Objective: To report the nutritional status and efficacy of nutritional support in patients with brain tumors that developed the Russell's Syndrome. Method: Seven patients were retrospectively evaluated by means of z-score of the weight for age (W/A), weight for height $(\mathrm{W} / \mathrm{H})$ and height for age $(\mathrm{H} / \mathrm{A})$ nutritional status index, for protein-energy malnutrition diagnosis. They were evaluated by means of triceps skinfold thickness (TSFT), arm circumferences (AC) and muscle arm circumferences $(\mathrm{MAC})$ and received enteral nutrition, by nasoenteral tube or gastrostomy at cancer diagnostic. Results: The ages ranged from 2 months to 13 years, five children were males. Mean of the nutritional support was 7 months (1.1-18.5 months) after diagnostic, without statistical differences in z-scores evolution, but there are increase in averages of the W/A $(-4,42$ to $-3,50)$ and $\mathrm{W} / \mathrm{H}(-3,06$ to $-1,99)$, and body composition indicators (TSFT): 2.85 to 4.88, AC: 9.81 to 11.84 and MAC: 8.91 to 10.31 ). There was decreased in average of $\mathrm{H} / \mathrm{A}$, evidencing the growth arrest of these children. Conclusion: Enteral feeding has been shown to guarantee nutritional supply and to partially recover nutritional indicators of acute malnutrition; especially body fat, rather than lean mass. However, the growth deficit was not corrected, being aggravated in most cases. Nutritional support should be implanted during oncological treatment, ensuring its maintenance.

Key words: Silver-Russell Syndrome; Brain Neoplasms; Enteral Nutrition; Nutrition Therapy; Child.
\end{abstract}

Resumen

Introducción: El síndrome diencefalica es una enfermedad pediátrica rara, derivada de tumores de la región hipotalámica, caracterizada por failure to thrive. Objetivo: Describir condiciones nutricionales y terapia nutricional de pacientes con tumores cerebrales com síndrome diencefalica y nutrición enteral. Método: Siete pacientes fueron acompańados de julio/1999 a abril/2002 y analizados retrospectivamente, usando el score- $Z$ de peso para edad $(\mathrm{P} / \mathrm{I})$, peso para estatura $(\mathrm{P} / \mathrm{E})$ y estatura para edad $(\mathrm{E} / \mathrm{I})$ para el diagnóstico de la desnutrición. Todos fueron evaluados por composición corporal (pliegue cutáneo triciptal y circunferencias del brazo y muscular del brazo). Los pacientes recibieron nutrición enteral por sonda o gastrostomía, luego del diagnóstico de cancer. Resultados: La edad varía de 2 meses a 13 ańos, cinco del sexo masculino. La duración media de la nutrición enteral fue de 7 meses $(1,1-18,5)$ después del diagnóstico. No hubo diferencia estadística en la evolución nutricional, a pesar del aumento en P/I (-4,42 a -3,50) y P/E (-3,06 a -1,99), así como en la composición corporal (PCT: 2,85 a 4,88, CB: 9,81 a 11,84 y CMB: 8,91 para 10.31). Hubo una reducción de E/I, caracterizando el retraso en el crecimiento. Conclusión: La nutrición enteral demostró garantizar la oferta nutricional.e la recuperación parcial de la desnutrición aguda, principalmente grasa corporal, más que masa magra, sin respuesta al déficit en el crecimiento, que se agravó en casi todos los casos. La terapia nutricional debe ser implantada durante el tratamiento oncológico, asegurando su continuidad.

Palabras clave: Síndrome de Silver-Russell; Neoplasias Encefálicas; Nutrición Enteral; Terapia Nutricional; Niño.

\footnotetext{
${ }^{1}$ Instituto de Oncologia Pediátrica (IOP). Grupo de Apoio ao Adolescente e à Criança com Câncer (Graacc). Universidade Federal de São Paulo (Unifesp). Orcid iD: https://orcid.org/0000-0001-7703-7088

${ }^{2}$ IOP-Graacc/Unifesp. São Paulo (SP), Brasil. Orcid iD: https://orcid.org/0000-0002-7759-9125

${ }^{3}$ IOP-Graacc/Unifesp. São Paulo (SP), Brasil. Orcid iD: https://orcid.org/0000-0002-9750-0508

Endereço para correspondência: Adriana Garófolo. Rua Manoel da Nóbrega, 1088, apto. 131 - Paraíso. São Paulo (SP), Brasil. CEP 04001-000.

E-mail: adrianagarofolo@graacc.org.br.
} 


\section{INTRODUÇÃO}

Tumores do sistema nervoso central são sólidos, comuns na idade pediátrica, correspondendo à segunda causa de neoplasias malignas na infância ${ }^{1}$. Desses, $40-$ $45 \%$ são gliomas de baixo grau, que são tumores das células gliais. A síndrome diencefálica é uma doença neurológica pediátrica rara, provocada por gliomas, quando de origem hipotalâmica anterior ou de quiasma óptico ${ }^{2}$. Podem ocorrer alterações de balanço energético, hormonais, hidroeletrolíticas, entre outras, desencadeando distúrbios nutricionais importantes, com consequente failure to thrive, como insuficiência de crescimento e magreza anormal (emagrecimento acentuado). A síndrome diencefálica é comumente observada na primeira infância, com poucos relatos em crianças maiores e adultos ${ }^{3-5}$.

A caquexia é uma condição frequentemente observada em bebês e crianças menores com síndrome diencefálica. Alteraçôes no apetite, gasto energético e absorção de nutrientes são responsáveis pelo déficit nutricional, predispondo à síndrome de realimentação ${ }^{3,4}$.

Pacientes com tumores cerebrais sob quimioterapia intensiva necessitam de terapia nutricional por período prolongado, com preferência à nutrição enteral, quando comparados a outros diagnósticos ${ }^{6,7,8}$.

Estudos avaliando novas propostas para desnutrição em crianças com câncer são importantes, haja vista seu impacto na tolerância ao tratamento, resposta à quimioterapia e tempo de sobrevida? ${ }^{9}$. Nos últimos anos, métodos de terapia nutricional nesse grupo têm sido discutidos ${ }^{10}$. Entre os pacientes com câncer, que se beneficiam de terapia nutricional, estáo crianças com tumores cerebrais, particularmente aquelas com a síndrome diencefálica ou síndrome de Russell.

O objetivo deste estudo é descrever a evolução do estado nutricional e a terapia nutricional por meio da nutrição enteral de crianças com síndrome diencefálica.

\section{MÉTODO}

Trata-se de uma análise retrospectiva de série de casos, com dados coletados de evoluções clínicas e nutricionais de sete crianças com tumores cerebrais, que desenvolveram a síndrome diencefálica, realizada no Instituto de Oncologia Pediátrica/Grupo de Apoio ao Adolescente e à Criança com Câncer/Universidade Federal de São Paulo (IOP/ Graacc/Unifesp), Brasil.

Os pacientes foram tratados e acompanhados de forma protocolada de julho de 1999 a abril de 2002. A coleta dos dados foi efetuada do diagnóstico até a última consulta nutricional, documentada em registros e planilhas eletrônicas de cada paciente. O tempo de acompanhamento nutricional variou de acordo com o tratamento oncológico proposto e sobrevida do paciente.

Foram incluídos todos os portadores de tumores cerebrais com a síndrome diencefálica com confirmação diagnóstica no período do acompanhamento. Os critérios de exclusão foram tumores cerebrais que não desenvolveram a síndrome diencefálica.

Os escores-z de P/E, P/I e E/I foram aplicados ao diagnóstico e na última avaliação e classificados de acordo com a Organizaçấo Mundial da Saúde $(\mathrm{OMS})^{11}$, que era a metodologia usada na época do acompanhamento. As espessuras da prega cutânea tricipital (PCT), da circunferência do braço $(\mathrm{CB})$ e da circunferência muscular do braço (CMB) foram obtidas ao mesmo tempo. A PCT foi determinada por meio do adipômetro (modelo Harpenden/Cescorf) e a CMB aferida no ponto médio entre acrômio e olécrano e calculada pela equação: $A M C=$ MUAC $-($ TSFT $x$ 0,314). Essas variáveis foram aferidas e interpretadas de acordo com padrōes e tabelas de percentis ${ }^{12}$.

A alimentação foi administrada por sonda enteral ou gastrostomia (laparoscópica ou cirúrgica) ao diagnóstico da neoplasia. Os pacientes receberam fórmulas industrializadas poliméricas e, no caso de má absorção e/ ou diarreia durante o estudo, fórmulas oligoméricas (fácil absorção) foram ofertadas.

Análise descritiva das variáveis demográficas, do estado nutricional e terapia nutricional foram demonstradas como médias e desvios-padrão.

Para a análise das diferenças entre as avaliaçôes, após intervençáo, foi aplicado o teste de postos de Wilcoxon. O nível de significância estabelecido foi menor ou igual a 0,05 ou $5 \%$.

O Comitê de Ética Médica da Escola Paulista de Medicina da Unifesp aprovou este estudo, sob o número de protocolo CEP 1792/07 (21/12/2007), garantindo os direitos de privacidade dos pacientes, de acordo com o Código de Ética da Associação Médica Mundial (Declaração de Helsinque) para estudos envolvendo seres humanos.

\section{RESULTADOS}

A idade variou de dois meses a 13 anos; sete pacientes foram acompanhados, cinco eram do sexo masculino. A média de duração da terapia nutricional foi 6,9 meses (mediana 2,8 meses; 1,1-18,5) após o diagnóstico. Um paciente (número 6) manteve o seguimento após recidiva do tumor. Cinco pacientes necessitaram de dietas oligoméricas desde o início.

Cinco crianças receberam quimioterapia e seis realizaram cirurgia. Não foram observadas complicações 
gastrintestinais ou infecciosas associadas à nutrição enteral. Seis pacientes evoluíram para óbito, três por complicaçôes pós-cirúrgicas relacionadas ao tratamento oncológico e três por progressão da doença, diagnosticada após a última avaliaçáo nutricional, no decorrer do tratamento.

Levando-se em conta o crescimento, quatro crianças tinham déficit de estatura grave e duas, déficit de estatura moderado ao diagnóstico. Após a terapia nutricional, seis delas apresentaram déficit de estatura; cinco, déficit grave; e uma, déficit moderado. Das seis crianças com comprometimento estatural, somente uma não demonstrou piora e apenas uma não tinha déficit de estatura durante o tratamento.

Quanto à adequação de peso, inicialmente três apresentaram déficit moderado e quatro, grave; e, ao final, uma era desnutrida moderada e quatro, grave. As médias dos escores-z de peso aumentaram da $1^{\circ}$ para a última avaliação $(\mathrm{P} / \mathrm{I}=-4,42$ para $-3,50 ; \mathrm{P} / \mathrm{E}=-3,06$ para $-1,99)$, porém sem diferença significante entre os períodos (Tabela 1). Os valores médios referentes às massas gorda e magra demonstraram aumentar (Tabela 2), também sem diferenças significantes.

O tempo de terapia nutricional variou entre os pacientes, com duração de 26 dias a 18,5 meses, como descrito na Tabela 1 . A nutrição parenteral total não foi administrada como terapia adjuvante na recuperação do estado nutricional e também não foi necessária de forma exclusiva por complicações gastrointestinais. Os pacientes de número 4, 5 e 7 náo conseguiram realizar a terapia nutricional por tempo mais prolongado, em razão do óbito precoce no período pós-operatório.

Tabela 1. Distribuição dos artigos segundo autores, título, periódico, população/participantes; metodologia, tamanho da amostra e principais resultados, 2003- 2018

\begin{tabular}{|c|c|c|c|c|c|c|c|c|c|c|c|c|c|c|c|}
\hline $\mathbf{P}$ & SN & $\begin{array}{l}\text { Idade } \\
\text { meses }\end{array}$ & SN & $\mathrm{Pi}$ & Pf & $\mathbf{E} \mathbf{i}$ & Ef & EzP/I i & EzP/I f & $\mathrm{EzE} / \mathrm{i}$ & $E z E / I f$ & EzP/E i & EzP/E f & EN i & EN $f$ \\
\hline 1 & SNE/SNG/GT & 6,39 & 18,5 & 4,79 & 10,14 & 62 & 78 & $-3,13$ & $-1,90$ & $-2,17$ & $-3,08$ & $-2,15$ & $-0,45$ & DEM/DPM & DEG \\
\hline 2 & SNE/GT & 24,03 & 13,9 & 7,08 & 13,8 & 74 & 79,8 & $-4,10$ & $-0,52$ & $-3,48$ & $-4,25$ & $-2,39$ & 2,33 & DEG/DPM & DEG/ DPM \\
\hline 3 & SNE/SNG & 10,32 & 9,6 & 4,00 & 6,43 & 62,5 & 72 & $-5,65$ & $-4,33$ & $-4,21$ & $-3,65$ & $-3,51$ & $-3,44$ & DEG/ DPG & DEG/DPG \\
\hline 4 & SNG & 8 & 1,6 & 3,00 & 3,08 & 57,3 & 58,4 & $-5,99$ & $-6,29$ & $-5,15$ & $-5,27$ & $-3,23$ & $-3,46$ & DEG/DPG & DEG/DPG \\
\hline 5 & SNG/SNE & 10,81 & 1,1 & 4,17 & 4,28 & 63 & 63 & $-4,96$ & $-5,01$ & $-3,60$ & $-3,99$ & $-3,29$ & $-3,12$ & DEG/ DPG & DEG/DPG \\
\hline 6 & SNG/VO & 163 & 2,8 & 17,3 & 19,0 & 136,5 & 137 & $-4,03$ & $-3,93$ & $-2,80$ & $-2,92$ & $-4,75$ & $-4,25$ & DEM/ DPG & DEM/DPG \\
\hline 7 & SNG/SNE & 4,68 & 1,8 & 5,00 & 5,99 & 63 & 65 & $-3,11$ & $-2,53$ & $-1,77$ & $-1,66$ & $-2,11$ & $-1,51$ & DPM & SDMG \\
\hline$M$ & & 32,46 & 6,9 & & & & & $-4,42$ & $-3,50$ & $-3,31$ & $-3,55$ & $-3,06$ & $-1,99$ & - & - \\
\hline $\mathrm{DP}$ & & 57,91 & & & & & & 1,15 & 1,97 & 1,17 & 1,14 & 0,94 & 2,31 & - & - \\
\hline \multicolumn{2}{|c|}{ Análise estatística } & $\begin{array}{l}\text { Teste de } \\
\text { Wilcoxon }\end{array}$ & & & & & & \multicolumn{2}{|c|}{$\begin{array}{c}p=0,0 \\
N S\end{array}$} & \multicolumn{2}{|c|}{$\begin{array}{c}p=0,18 \\
\text { NS }\end{array}$} & \multicolumn{2}{|c|}{$\begin{array}{c}p=0,06 \\
\text { NS }\end{array}$} & & \\
\hline
\end{tabular}

Legendas: $\mathrm{P}$ = peso; i = inicial; f: final; SN (em meses): suporte nutricional; SN: tempo ou período de suporte nutricional; SNG: sonda nasogástrica; SNE: sonda nasoenteral; EN: estado nutricional; DEM: déficit estatural moderado; DEG: déficit estatural grave; DPM: déficit ponderal moderado; DPG: déficit ponderal grave; SDMG: sem déficit moderado ou grave de acordo com os escores-z de P/E e E/I; M = média; DP = desvio-padrão; NS = não significante.

Tabela 2. Descrição dos sete pacientes quanto às variáveis de composição corporal (média e desvio-padrão) antes e após intervenção com sonda ou gastrostomia no IOP/Graacc/Unifesp

\begin{tabular}{|c|c|c|c|c|c|c|c|c|}
\hline \multicolumn{2}{|l|}{ Paciente } & PCT i & PCT f & CB i & CB f & CMB i & CMB f & TSN \\
\hline \multicolumn{2}{|l|}{1} & 3,36 & 11,30 & 11,10 & 16,60 & 10,04 & 13,05 & 18,49 \\
\hline \multicolumn{2}{|l|}{2} & 4,30 & 9,80 & 11,00 & 16,50 & 9,63 & 13,42 & 13,96 \\
\hline \multicolumn{2}{|l|}{3} & 1,93 & 4,70 & 7,80 & 11,50 & 7,19 & 10,02 & 9,63 \\
\hline \multicolumn{2}{|l|}{4} & 2,56 & 2,33 & 7,50 & 7,20 & 6,69 & 6,46 & 1,60 \\
\hline \multicolumn{2}{|l|}{5} & 1,53 & 1,70 & 7,30 & 7,60 & 6,81 & 7,06 & 1,08 \\
\hline \multicolumn{2}{|l|}{6} & 2,86 & 1,60 & 13,50 & 13,00 & 12,60 & 12,49 & 2,81 \\
\hline \multicolumn{2}{|l|}{7} & 3,40 & 2,70 & 10,50 & 10,50 & 9,43 & 9,66 & 0,85 \\
\hline \multicolumn{2}{|l|}{ Média } & 2,85 & 4,88 & 9,81 & 11,84 & 8,91 & 10,31 & 6,92 \\
\hline \multicolumn{2}{|l|}{ Desvio-padrão } & 0,94 & 4,03 & 2,34 & 3,81 & 2,16 & 2,82 & 7,15 \\
\hline \multicolumn{2}{|l|}{ Mediana } & 2,86 & 2,70 & 10,5 & 11,50 & 9,43 & 10,02 & 2,81 \\
\hline Análise estatística & $\begin{array}{l}\text { Teste de } \\
\text { Wilcoxon }\end{array}$ & \multicolumn{2}{|c|}{$\begin{array}{c}p=0,09 \\
\text { NS }\end{array}$} & \multicolumn{2}{|c|}{$\begin{array}{c}p=0,18 \\
N S\end{array}$} & \multicolumn{2}{|c|}{$\begin{array}{c}p=0,06 \\
N S\end{array}$} & \\
\hline
\end{tabular}

Legendas: $\mathrm{i}$ = inicial; f: final; TSN: tempo de suporte nutricional em meses; PCT $(\mathrm{mm})$ : prega cutânea tricipital; $\mathrm{CB}(\mathrm{cm})$ : circunferência do braço; CMB (cm): circunferência muscular do braço; NS = não significante. 


\section{DISCUSSÃO}

O tratamento dos gliomas de baixo grau em idade pediátrica tem como "padrão-ouro" a remoção cirúrgica total, que se associa à maior sobrevida livre de doença em crianças. No entanto, nos casos em que o glioma não pode ser totalmente removido, o tratamento fica limitado à radioterapia ou quimioterapia. Por causa das altas taxas de progressão de doença em crianças com gliomas de baixo grau inoperáveis, muitas ficam sujeitas a tratamento clínico prolongado. A radioterapia resulta em uma diminuição objetiva em alguns pacientes e pode melhorar a sobrevida livre de progressão em crianças com tumores parcialmente ressecados, entretanto muitas crianças portadoras de gliomas hipotalâmicos/quiasmáticos de baixo grau são muito pequenas e esses gliomas geralmente de grande extensão. A radioterapia nessas circunstâncias pode causar sequelas neurológicas/cognitivas significantes. Entretanto, crianças muito pequenas com glioma de baixo grau têm uma sobrevida ruim. Tumores mutados por BRAF apresentaram resposta fraca à quimioterapia tradicional e prognóstico desfavorável. Em glioma pediátrico de baixo grau, uma mutação BRAFV600E foi identificada e, por esse motivo, a terapia-alvo tem sido investigada, apresentando sucesso em alguns desses casos. Tal condição leva as equipes médicas a encararem o glioma de baixo grau como uma doença crônica, baseando o tratamento no equilíbrio entre seus benefícios e morbimortalidade acarretada por ele ${ }^{13}$.

Crianças com síndrome diencefálica são propensas à grave déficit nutricional ao diagnóstico da neoplasia, com consequente failure to thrive, como observado neste estudo. A perda de gordura subcutânea com grave emagrecimento e retardo do crescimento, observados nesses pacientes, é comum. Como citado, a redução ou a dificuldade para ganhar peso podem ocorrer por diminuiçáo do apetite, bem como pelo hipermetabolismo. Além disso, distúrbios metabólicos somados a complicaçóes dos procedimentos terapêuticos dificultam a intervenção nutricional ${ }^{3}$.

O protocolo de tratamento para menores de cinco anos com síndrome diencefálica é a quimioterapia e a cirurgia, principalmente para descompressão tumoral ${ }^{5}$. Entretanto, a cirurgia é um procedimento extremamente agressivo, principalmente na presença de desnutrição grave. Assim, antes de submeter as crianças à agressão cirúrgica, recuperar o déficit nutricional torna-se fundamental, haja vista o impacto negativo da desnutriçáo na resposta perioperatória ${ }^{14}$. Neste estudo, as três crianças $(4,5$ e 7$)$ submetidas à cirurgia, logo após o diagnóstico, apresentavam comprometimento nutricional grave e náo sobreviveram.

A terapia nutricional deve levar em conta toxicidades gastrintestinais decorrentes da quimioterapia que, embora de graus mais leves nesses casos, contribuem para a desnutrição. Entre os principais efeitos adversos, náuseas, vômitos e constipação são os mais comuns, por conta dos tipos de medicamentos utilizados ${ }^{15}$.

No estudo, em razão do grau de desnutrição, baixa aceitação oral e sequelas da doença e tratamento, as vias de alimentação escolhidas foram sonda enteral e gastrostomia. A terapia nutricional faz parte do plano terapêutico nesses casos, com finalidade de manter os pacientes por período prolongado sob quimioterapia. Uma terapia nutricional eficiente garante a recuperaçáo e a manutenção do estado nutricional adequado e, portanto, o tratamento antineoplásico.

Embora a terapia nutricional seja fundamental, ao instituí-la, deve-se ter atenção aos riscos da síndrome diencefálica de realimentação, pela gravidade da desnutrição e inanição das crianças. As principais manifestaçôes são: distúrbio hídrico, deficiência de tiamina, hipofosfatemia, hipomagnesemia, hipocalemia e anormalidades no metabolismo da glicose ${ }^{16}$. Sua prevenção baseia-se na correção dos distúrbios hidroeletrolíticos e deficiência polivitamínica e mineral antes de começar a terapia nutricional, a ser inicialmente instituída com $50 \%$ das necessidades energéticas, progredindo gradativamente ${ }^{17}$.

A nutrição enteral é uma conduta bem estabelecida em pacientes pediátricos com câncer com algum grau de risco nutricional ou desnutrição, considerando que sua utilização está associada à recuperação do estado nutricional nessa população. Entretanto, ainda são necessários ensaios clínicos, avaliando seus benefícios na resposta terapêutica, incidência de complicaçôes e prognóstico ${ }^{18-21,2,22,23}$.

Dos sete pacientes do estudo, três receberam terapia nutricional por menos de dois meses (óbito no pós-operatório) e tiveram maior número de complicaçóes associadas à doença, o que prejudicou a oferta nutricional plena. Provavelmente, o curto período de terapia nutricional, somado às complicações clínicas, prejudicou a evolução nutricional.

Apesar de não haver diferença estatística na evolução nutricional do grupo, quatro crianças que receberam terapia nutricional acima de dois meses apresentaram aumento nos indicadores nutricionais.

Estudos de terapia nutricional em pacientes pediátricos com tumores cerebrais que desenvolvem a síndrome diencefálica não foram encontrados. Algumas evidências mostram que as células malignas do glioma dependem criticamente da glicose como principal fonte de energia para sobreviver e sustentar suas propriedades agressivas. Como a maioria dos cânceres, os glioblastomas geralmente têm mitocôndrias desreguladas, impedindo a eficiência 
do ciclo do ácido tricarboxílico e as atividades de fosforilação oxidativa, necessárias para a produção aeróbia de energia. A maioria das células tumorais depende da geração de energia por meio da via da glicólise anaeróbia, comparativamente ineficiente. Várias alteraçóes genéticas importantes encontradas em gliomas de alto grau relacionam o metabolismo energético ao câncer ${ }^{24}$. O conhecimento crescente sobre aberraçóes moleculares e genéticas nesses tumores sugere uma série de novas estratégias nutricionais, porém as terapias nutricionais propostas até o momento apresentam sucesso limitado. Existem poucas evidências e ainda pouco conclusivas, o que se deve, pelo menos em parte, à heterogeneidade molecular desses tumores. A dieta cetogênica tem sido testada para portadores de tumores cerebrais, porém necessita de mais estudos ${ }^{25}$.

Assim, a terapia nutricional convencional ainda é a conduta padrão. Em geral, os estudos realizados em crianças desnutridas com câncer que receberam terapia nutricional por meio de sonda enteral e gastrostomia com dietas hipercalóricas e hiperproteicas apresentam resultados positivos na recuperação do estado nutricional. Apesar disso, alguns autores relataram a necessidade de nutrição parenteral total adjuvante à nutrição enteral ${ }^{7,8,26}$.

Neste estudo, não se utilizou nutrição parenteral total, nem mesmo como terapia nutricional adjuvante para recuperação nutricional. Essa modalidade, como coadjuvante da nutrição enteral, não fez parte do planejamento terapêutico neste trabalho, pois sua utilização fica reservada para casos mais graves, cuja alimentação pelo trato gastrintestinal está prejudicada por toxicidade gastrintestinal grave ou contraindicada. Além disso, a ausência de protocolo para nutriçáo parenteral total no âmbito ambulatorial também não permitiu sua introdução. No entanto, nos casos em que a nutrição enteral náo foi capaz de contemplar as necessidades, a nutrição parenteral total combinada poderia melhorar os resultados do estado nutricional.

Métodos alternativos para crianças com câncer, cuja terapia nutricional necessita período prolongado, têm sido propostos. Nos últimos anos, a alimentação por gastrostomia vem substituindo as sondas enterais, pelas vantagens no uso prolongado. Além de outros aspectos, apresenta maior facilidade de manipulação pelos profissionais, pacientes e familiares, é esteticamente mais aceita e possui menos inconvenientes durante a quimioterapia, em razão das perdas e repassagem da sonda enteral por vômitos.

Resultados do estudo retrospectivo de Mathew et al., desenvolvido no St. Jude Children's Research Hospital, confirmam os dados com o uso da gastrostomia por período prolongado em crianças com câncer, sem complicaçôes graves e mortalidade pós-cirúrgica associada ao procedimento ${ }^{27}$. Da mesma forma, outros autores demonstraram a eficácia da gastrostomia endoscópica ou radiológica percutânea na manutenção ou recuperação nutricional, sendo factível em pacientes pediátricos oncológicos, com baixa taxa de complicaçôes graves. No entanto, a técnica por cirurgia aberta apresenta mais complicaçóes ${ }^{28-30}$.

No estudo atual, o déficit estatural foi mais acentuado no decorrer do acompanhamento. Embora todos os indicadores de composição corporal tenham aumentado após a terapia nutricional, independentemente do tipo de dispositivo (sonda enteral ou gastrostomia), houve recuperação do estado nutricional pela aquisição do peso, sem melhora da estatura. Esses resultados desfavoráveis quanto à estatura (Tabela 1), caracterizam o atraso no crescimento, característico do grupo. Tal fator associa-se, provavelmente, à condição de cronicidade da doença, que aumenta o catabolismo muscular. Por isso, a terapia nutricional não é capaz de modificar esse perfil nessa fase precoce. Entretanto, mesmo os pacientes que receberem a terapia nutricional por tempo maior (em duas crianças $>12$ meses) apresentaram esse déficit. Um fator limitante do estudo foi o caráter retrospectivo, com tempo de uso de terapia nutricional apresentando variação entre os pacientes (média 7; mediana 2,8 meses). Considerando as complicaçôes e intercorrências da doença e tratamento, essas crianças necessitam de um período prolongado para recuperaçáo do estado nutricional. Mas, não se sabe se o déficit seria maior, caso a terapia nutricional não fosse aplicada. Períodos curtos de suporte nutricional não permitem que as necessidades nutricionais sejam contempladas, o que interfere nos resultados do crescimento e desenvolvimento ${ }^{31}$. Além disso, como a doença é grave e a proposta terapêutica é em longo prazo, a terapia nutricional é uma condição fundamental para o maior sucesso do plano terapêutico.

Os resultados deste estudo foram similares aos do estudo de Skolin et al. ${ }^{29}$, que observaram atraso no crescimento linear de crianças com câncer após um ano de terapia nutricional que, segundo eles, poderia estar associado à gravidade da doença.

Três, das sete crianças, corrigiram o déficit ponderal grave; com uma tornando-se eutrófica, pois as demais evoluíram para sobrepeso e desnutrição leve. A evolução das médias mostra que a recuperaçáo por meio do $\mathrm{P} / \mathrm{E}$ foi a mais importante $(-3,06$ para $-1,99 ; \mathrm{p}=0,06)$. As duas crianças que usaram gastrostomia (pacientes 1 e 2) foram as que tiveram diagnóstico de eutrofia e sobrepeso, resultado provavelmente associado ao maior tempo de terapia nutricional (Tabela 1 ).

O excesso de ganho ponderal em longo prazo, principalmente caracterizado pelo aumento do tecido 
adiposo, configura-se como um fator de risco, comum em crianças com tumores cerebrais, quando a doença se torna crônica. Os indicadores de adiposidade (PCT) e de tecido muscular (CMB) demonstraram evolução similar (Tabela 2) e acompanharam os resultados do indicador de peso, embora não tenham demonstrado diferenças estatísticas.

Enquanto o pequeno número da amostra não permitiu encontrar diferenças significantes, os resultados individuais demonstram a importância da terapia nutricional para pacientes com síndrome diencefálica, e que ela é fundamental para recuperar, pelo menos em parte, o déficit nutricional dessas crianças. Por outro lado, comprometimento no crescimento e no desenvolvimento e risco de acúmulo de gordura são sequelas importantes em longo prazo durante o acompanhamento.

Atualmente, o tratamento antineoplásico é efetuado com quimioterapia por tempo prolongado para permitir a intervenção cirúrgica mais tardia e com maior sucesso e menor risco de complicaçóes, o que exige apoio nutricional também por período prolongado.

\section{CONCLUSÃO}

Crianças com síndrome diencefálica devem ser tratadas como pacientes crônicos, para os quais o planejamento terapêutico deve ser efetuado de forma cautelosa, por meio da assistência multidisciplinar, reduzindo-se a morbimortalidade. A desnutrição grave é uma das características iniciais da síndrome diencefálica, tornando o tratamento da doença um desafio ainda maior para a equipe. A terapia nutricional possibilitou recuperação parcial do estado nutricional, principalmente com o uso mais prolongado. A gastrostomia percutânea pode ser um método mais eficiente para garantir o aporte energético e nutricional e assegurar o tratamento por período maior, aumentando a oportunidade de recuperação nutricional e sucesso na resposta antineoplásica ${ }^{32}$.

Ajustes na oferta nutricional devem ser efetuados ao longo do seguimento, com cuidados visando a sequelas no crescimento e desenvolvimento e ao risco de aumento excessivo do tecido adiposo em longo prazo.

\section{CONTRIBUIÇÕES}

Os autores contribuíram igualmente em todas as etapas do manuscrito.

\section{DECLARAÇÃO DE CONFLITO DE INTERESSES}

Nada a declarar.

\section{FONTES DE FINANCIAMENTO}

Não há.

\section{REFERÊNCIAS}

1. Instituto Nacional de Câncer José Alencar Gomes da Silva. Estimativa 2018: incidência de câncer no Brasil. Rio de Janeiro: INCA; 2017.

2. Groupman AL, Packer RJ, Nicholson HS, Vezina LG, Jakacki R, Geyer R, et al. Treatment of diencephalic syndrome with chemotherapy. Cancer. 1998 Jul 83;(1):166-172.

3. Waga S, Shimizu T, Sakakura M. Diencephalic syndrome of emaciation (Russell's syndrome). Surg Neurol. 1982 Feb;17(2):141-146.

4. Espiner E, Donaldson I, Chapman B, Bergeron C. Diencephalic idiopathic gliosis: an unusual hypothalamic syndrome of dermopathy, diarrhea and growth arrest. J Endocrinol Invest. 1992 Sep;15(8):609-616.

5. West GA, Berger MS, Geyer JR. Childhood optic pathway tumors associated with ascites following ventriculoperitoneal shunt placement. (case report) Pediatr Neurosurg 1994 Mar;21(4):254-259; discussion 259.

6. Tyc VL, Vallelunga L, Mahoney S, Smith BF, Mulhern RK. Nutritional and treatment-related characteristics of pediatric oncology patients referred or not referred for nutritional support. Med Pediatr Oncol. 1995 Nov;25(5):379-388.

7. Sanchez MC, Iraola GA, Gutierrez NA, Altuna MS, Regato JLB. Estudio nutricional en niños oncológicos. An Esp Pediatr. 1992;36(4):277-280.

8. Pietsch JB, Ford CR, Whitlock JA. Nasogastric tube feeding in children with high-risk cancer: a pilot study. J Pediatr Hematol Oncol. 1999 Mar-Apr;21(2):111-114.

9. Pribnow AK, Ortiz R, Báez LF, Mendieta, L, LunaFineman S. Effects of malnutrition on treatmentrelated morbidity and survival of children with cancer in Nicaragua. Pediatr Blood Cancer 2017 Nov;64(11). doi:10.1002/pbc.26590. Epub 2017 Apr 27.

10. Steele C, Salazar A, Rypkema L. Utilization of a nutrition support algorithm reduces unnecessary parenteral nutrition use in pediatric oncology inpatients. J Acad Nutr Diet 2016 Aug;116(8):1235-128. doi:10.1016/j. jand.2015.12.007. Epub 2016 Jan 28.

11. Management of severe malnutrition: a manual for physicians and other senior health workers. World Health Organization: Geneva; 1999.

12. Frisancho AR. Anthropometric standards for the assessment of growth and nutritional status. 4nd ed. Ann Arbor: University of Michigan Press; 1993.

13. Martins AR. Terapêutica dos Gliomas de Baixo Grau em Idade Pediátrica [dissertação]. Lisboa. Clínica 
Universitária de Neurocirurgia, Faculdade de Medicina, Universidade de Lisboa; 2017.

14. Windsor JA, Graham LH. Weight loss with physiologic impairment: a basic indicator of surgical risk. Ann Surg 1988 Mar; 207(3):290-296.

15. Nurgali K, Jagoe RT, Abalo R. Editorial: adverse effects of cancer chemotherapy: anything new to improve tolerance and reduce sequelae?. Front Pharmacol. 2018 Mar 22;9:245. doi: 10.3389/fphar.2018.00245.

16. Crook MA, Hally V, Panteli JV. The importance of the refeeding syndrome. Nutrition. 2001 JulAug;17(7-8):632-637.

17. Afzal NA, Addai S, Fagbemi A, Murch S, Thomson M, Heuschkel R. Refeeding syndrome with enteral nutrition in children: a case report, literature review and clinical guidelines. Clin Nutr 2002 Dec;21(6):515-520.

18. Bowman LC, Williams R, Sanders M, Ringwald-Smith K, Baker D, Gajjar A. Algorithm for nutritional support: experience of the metabolic and infusion support service of St. Jude Children's Research Hospital. Int J Cancer. 1998;78(11 Suppl):76-80.

19. Pencharz PB. Aggressive oral, enteral or parenteral nutrition: prescriptive decisions in children with cancer. Int J Cancer. 1998;78(11 Suppl):73-75.

20. den Broeder E, Lippens RJ, van't Hof MA, Tolboom JJ, Sengers RC, van den Berg AM, et al. Nasogastric tube feeding in children with cancer: the effect of two different formulas on weight, body composition, and serum protein concentrations. JPEN J Parenter Enteral Nutr 2000 Nov;24(6):351-360.

21. Heubi JE. Whenever possible, use the gut! J Pediatr Hematol Oncol 1999 Mar-Apr; 21(2):88-90.

22. Hastings Y, White M, Young J. Enteral nutrition and bone marrow transplantation. J Pediatr Oncol Nurs 2006 Mar-Apr;23(2):103-110.

23. Broeder E, Lippens RJJ, Van't Hof MA, Tolboom J. Effects of naso-gastric tube feeding, on the nutritional status of children with cancer. Eur J Clin Nutr 1998 Jun;52(7):494-500.

24. Woolf EC, Syed N, Scheck AC. Tumor metabolism, the ketogenic diet and $\beta$-Hydroxybutyrate: novel approaches to adjuvant brain tumor therapy. Front Mol Neurosci. 2016 Nov 16;9:122. doi:https://doi.org/10.3389/ fnmol.2016.00122.

25. Winter SF, Loebel F, Dietrich J. Role of ketogenic metabolic therapy in malignant glioma: a systematic review. Crit Rev Oncol Hematol. 2017 Apr;1 12:41-58. doi:https://doi.org/10.1016/j.critrevonc.2017.02.016.

26. Deswarte-Wallace J, Firouzbakhsh S, Finklestein JZ. Using research to change practice: enteral feedings for pediatric oncology patients. J Pediatr Oncol Nurs. 2001 Sep 1;18(5):217-223.

27. Mathew P, Bowman L, Williams R, Jones D, Rao B, Schropp K, et al. Complications and effectiveness of gastrostomy feedings in pediatric cancer patients. J Pediatr Hematol Oncol. 1996 Feb;18(1):81-85.

28. Barron MA, Duncan DS, Green GJ, Modrusan D, Connolly B, Chait P, et al. Efficacy and safety of radiologically placed gastrostomy tubes in paediatric haematology/oncology patients. Med Pediatr Oncol 2000 Mar;34(3):177-182.

29. Skolin I, Hernell O, Larsson MV, Wahlgren C, Wahlin YB. Percutaneous endoscopic gastrostomy in children with malignant disease. J Pediatr Oncol Nurs 2002 Sep 1;19(5):154-163.

30. Fernandez-Pineda I, Sandoval JA, Jones RM, Boateng $\mathrm{N}, \mathrm{Wu}$ J, Rao BN, et al. Gastrostomy complications in pediatric cancer patients: a retrospective single-institution review. Pediatr Blood Cancer. 2016 Jul;63(7):1250-1253. doi: 10.1002/pbc.25968. Epub 2016 Mar 9.

31. Garófolo A, Maia PS, Petrilli AS, Ancona-Lopez F. Resultados da implantação de um algoritmo para terapia nutricional enteral em crianças e adolescentes com câncer. Rev. Nutr 2010 Set-Out;23(5):715-730.

32. Pederson AM, Kok K, Petersen G, Nielsen OH, Michaelsen KF, Schimiegelow K. Percutaneous endoscopic gastrostomy in children with cancer. Acta Paediatr 1999 Aug;88(8):849-852. 